\title{
Azithromycin verbessert Asthmasymptomatik
}

Gibson PG et al. Effect of azithromycin on asthma exacerbations and quality of life in adults with persistent uncontrolled asthma (AMAZES): a randomised, double-blind, placebo-controlled trial. Lancet 2017; 390: 659-668

Schon seit einiger Zeit gibt es Hinweise darauf, dass Makrolidantibiotika den Verlauf einer unzureichend kontrollierten Asthma-bronchiale-Erkrankung positiv beeinflussen können. Jetzt wurde in einer prospektiven Studie gezeigt, dass die Einnahme von Azithromycin die Exazerbationshäufigkeit senken kann.

In die randomisierte, Placebo-kontrollierte, doppelt-verblindete AMAZES-Studie wurden zwischen 2009 und 2015 insgesamt 420 Patienten mit Asthma bronchiale aufgenommen, die trotz der $\mathrm{Er}$ haltungstherapie mit einem inhalativen Kortikosteroid und einem langwirksamen Bronchodilatator symptomatisch waren. Ausschlusskriterien waren eine verringerte Diffusionskapazität durch Lungenparenchymveränderungen oder eine Nikotinanamnese $>10$ pack years bzw. ein aktueller Nikotinabusus. Außerdem wurden aufgrund des Nebenwirkungsspektrums von Azithromycin Patienten mit abnorm verlängerter QT-Zeit im Ruhe-EKG oder Beeinträchtigungen des Gehörs nicht in die Studie aufgenommen.

Die Aktivität der Asthmaerkrankung wurde mit dem ACQ6 (Asthma Control Score) erfasst - ein Fragebogen mit 6 Symptom-Items, die jeweils auf einer Skala von 0-6 bewertet werden mussten. Die Asthma-spezifische Lebensqualität wurde mit dem AQLQ-Fragebogen gemessen.

Es erfolgte eine 1:1-Randomisierung in eine Gruppe, die über einen Zeitraum von 48 Wochen zusätzlich zur inhalativen Dauertherapie 3-mal/Woche $500 \mathrm{mg}$ Azithromycin erhielt, und eine PlaceboGruppe.

Verlängerungen der korrigierten QT-Zeit $>480$ ms unter der Therapie führten zum Studienausschluss. Als primären Endpunkt definierten die Autoren das Auf- treten von Asthma-Exazerbationen und die asthmaspezifische Lebensqualität. Schwere Exazerbationen wurden dabei definiert als die Notwendigkeit einer zusätzlichen Behandlung mit systemischen Kortikosteroiden bzw. die Anhebung der systemischen Kortikosteroiddosis über einen Zeitraum von mindestens 3 Tagen oder eine notfallmäßige Akutvorstellung bzw. Hospitalisierung aufgrund des Asthma bronchiale. Die Patienten der AMAZES-Kohorte waren im Median 60 Jahre alt, bei $76 \%$ lag ein atopisches Asthma vor und die Diagnose war im Median seit 32 Jahren bekannt. Azithromycin führte im Vergleich zum Placebo zu einer $41 \%$ igen Reduktion der Exazerbationsrate. Auch die Häufigkeit von respiratorischen Infektionen lag in der Azithromycin-Gruppe signifikant niedriger als in der Placebo-Gruppe (17 vs. $31 \%$ ). Außerdem führte die Einnahme von Azithromycin zu einer Verbesserung der Lebensqualität. Als häufigste Nebenwirkung wurden unter der Azithromycineinnahme Diarrhöen verzeichnet (34 vs. $19 \%$ in der Placebo-Gruppe), die aber selten zu einem Behandlungsabbruch führten.

\section{FAZIT}

Die additive Gabe von Azithromycin zur inhalativen Dauertherapie erwies sich bei Patienten mit unzureichend kontrolliertem Asthma bronchiale als gut verträglich, senkte die Häufigkeit von akuten Exazerbationen und verbesserte die Lebensqualität der Patienten. Ob es unter einer solchen Behandlung zu einer klinisch und epidemiologisch relevanten Resistenzentwicklung kommt, sollte allerdings in weiteren Studien geklärt werden, so die Autoren.

Dr. Katharina Franke, Darmstadt

\section{Kommentar}

Die Basis der medikamentösen Therapie bei Patienten mit Asthma bronchiale besteht aus dem Einsatz eines inhalativen Steroides (ICS), ggf. in Kombination mit langwirksamen Bronchodilatatoren. Bei Patienten, die unter dieser Kombination nicht adäquat kontrolliert sind, stellt sich die Frage nach weiteren Therapieoptionen. Die Wirksamkeit von Makrolidantibiotika umfasst neben der antibiotischen Wirkung auch anti-entzündliche sowie anti-virale Effekte. Besonders in vitro lassen sich dabei anti-entzündliche Effekte von Makroliden auf klassische Charakteristika der asthmatischen Entzündungsreaktion nachweisen [1].

Der klinische Einsatz von Makrolidantibiotika bei Patienten mit Asthma wird schon seit längerer Zeit diskutiert. Ein Effekt einer Therapie mit Makrolidantibiotika bei Patienten mit leichtem Asthma oder während einer akuten Exazerbation konnte aber nicht nachgewiesen werden $[2,3]$. Anders sieht die Datenlage bei Patienten mit moderatem bis schwerem Asthma aus. Bisherige Studien ließen vermuten, dass durch den chronischen Einsatz von Makrolidantibiotika bei einigen Patienten mit Asthma eine Reduktion von Asthmaexazerbationen erreicht werden kann $[4,5]$. In diesen Untersuchungen war die Behandlung mit Azithromycin besonders bei Patienten ohne Nachweis einer Entzündungsreaktion mit eosinophilen Granulozyten wirksam. Die aktuelle Studie ist die bisher größte und längste Interventionsstudie, die den Effekt einer Behandlung mit Azithromycin bei Patienten mit Asthma untersucht hat. Die Daten zeigen, dass bei den Patienten durch die Behandlung mit Azithromycin eine Reduktion der akuten Exazerbationen erreicht werden kann. Der Effekt der Behandlung war aber nicht an spezifische Patientencharakteristika gebunden. Sowohl bei Patienten mit dem Nachweis einer erhöhten Anzahl an 
eosinophilen Granulozyten im Sputum als auch bei Patienten ohne eine solche Entzündungsreaktion konnte durch die Gabe von Azithromycin die Anzahl von Exazerbationen gesenkt werden. Diese Ergebnisse legen nahe, dass bei Patienten mit nicht-kontrolliertem Asthma unter inhalativer Behandlung die zusätzliche langfristige Gabe von Azithromycin sinnvoll ist und dieser Effekt unabhängig vom Asthmaphänotyp ist.

Dieser Ansatz kann aber nicht unkritisch in die Praxis überführt werden. Auffallend in der aktuellen Studie ist das Lebensalter der untersuchten Patienten. Diese waren in der aktuellen Untersuchung im Mittel 60 Jahre, wobei die Erkrankung bereits um das 20. Lebensjahr diagnostiziert worden war. Es handelt sich daher um eine ungewöhnlich alte Asthmapopulation mit sehr langer Krankheitsgeschichte. Zusätzlich waren beinahe $40 \%$ der Patienten Ex-Raucher mit gemittelt 7,5 pack years. Gerade bei Patienten mit einem seit langem bestehenden Asthma kann es zu nicht unerheblichen strukturellen Umbauprozessen in den Atemwegen kommen, die als Remodeling der Atemwege bezeichnet werden. Welche Auswirkungen diese Prozesse auf das Mikrobiom der Lunge oder die bakterielle Besiedelung der Atemwege haben, ist nicht deutlich. Die Ergebnisse der aktuellen Studie können aber daher nicht unkritisch auf Kinder oder junge Patienten mit Asthma extrapoliert werden.

Auch muss betont werden, dass eine langfristige Therapie mit Azithromycin einen Einfluss auf die bakterielle Resistenzentwicklung hat. In der vorliegenden Studie war nach Behandlung mit dem Makrolidantibiotikum eine Zunahme von Azithromycin-resistenten Organsimen im Sputum nachweisbar. Zusätzlich hat die Behandlung mit Azithromycin einen Effekt auf die Reizleitung im Herzen. Insbesondere Patienten mit einer verlängerten QT-Zeit oder kardialer Vorerkrankung sollten nicht mit Azithromycin behandelt werden. Für bestimmte Patientengruppen mit schwerem Asthma (z.B. schweres allergisches Asthma und schweres Asthma mit erhöhter Anzahl von eosinophilen Granulozyten im
Blut) stehen sichere Behandlungsoptionen in der Form von monoklonalen Antikörpern zur Verfügung.

Daher sollte Azithromycin nicht unkritisch als weitere Add-on-Behandlung bei Patienten mit Asthma eingesetzt werden. Der Gebrauch kann bei Patienten erwogen werden, die trotz adäquater, hochdosierter inhalativer Therapie häufig Exazerbationen haben und für die keine therapeutischen Alternativen zur Verfügung stehen. Es bleibt aber eine nicht unerhebliche Sorge über die weitere Entwicklung von Antibiotikaresistenzen durch die langfristige Gabe. Daher wäre die weitere Entwicklung von nicht antibiotisch, aber anti-entzündlich wirksamen Makrolidsubstanzen wünschenswert.

\section{Autorinnen/Autoren}

Prof. Dr. Christian Taube,

Klinik für Pneumologie,

Universitätsmedizin,

Essen-Ruhrlandklinik

\section{Literatur}

[1] Mertens TC, Hiemstra PS, Taube C. Azithromycin differentially affects the IL-13-induced expression profile in human bronchial epithelial cells. Pulm Pharmacol Ther 2016; 39: 14 20

[2] Sutherland ER, King TS, Icitovic $\mathrm{N}$ et al. A trial of clarithromycin for the treatment of suboptimally controlled asthma. J Allergy Clin Immunol 2010; 126: 747 - 753

[3] Johnston SL, Szigeti M, Cross M et al. Azithromycin for Acute Exacerbations of Asthma: The AZALEA Randomized Clinical Trial. JAMA int medi 2016; 176: 1630 - 1637

[4] Brusselle GG, Vanderstichele C, Jordens P et al. Azithromycin for prevention of exacerbations in severe asthma (AZISAST): a multicentre randomised double-blind placebocontrolled trial. Thorax 2013; 68: 322 - 329

[5] Kew KM, Undela K, Kotortsi I et al. Macrolides for chronic asthma. The Cochrane Rev 2015. doi:Cd002997 